Journal of Education and Vocational Research

Vol. 5, No. 4, pp. 191-197, December 2014 (ISSN 2221-2590)

\title{
Z-Theory, Foundation and Frame
}

\author{
Allan Zade \\ Russian Federation \\ AllanZadeUK@gmail.com
}

\begin{abstract}
Despite the fact that Z-Theory was published few years ago (2011), many questions arise until today about nature of the theory, its cause and reason. This article is dedicated to answering key questions of the theory, to lift the veil of "mystery" that surrounds the theory for many centuries and keeps the human mind incompatible with Z-Theory.
\end{abstract}

Keywords: Z-Theory, Philosophy, Logical Separator, Comprehension Horizon, Time, Space, Z-Trajectory, Transposition

\section{Introduction}

What is a scientific theory? That question can be discussed for ages leaving many aspects beyond the scope of the discussion. However, there is the following answer according the modern point of view. A scientific theory is a systematic ideational structure of broad scope, conceived by the human imagination that encompasses a family of empirical (experiential) laws regarding regularities existing in objects and events, both observed and posited. A scientific theory is a structure suggested by these laws and is devised to explain them in a scientifically rational manner ("Scientific," 2008, Para. 1). There is a very important aspect in a given definition. A scientific theory is "a structure... conceived by the human imagination". Suppose now that there are two philosophers (A and B) working on the same "family of empirical (experiential) laws regarding regularities existing in objects and events". However, philosopher "A" conceived those laws unlike philosopher "B" who does not conceive the same laws. As a result, philosopher " $\mathrm{A}$ " becomes able to produce a theory relevant to the "family of empirical (experiential) laws regarding regularities existing in objects and events". At the same time, philosopher "B" becomes unable to reach the same result because his/her imagination is unable to conceive the same regularities. More than that, it is quite possible for those philosophers to produce more than one theory according the way that each philosopher uses to conceive "those laws" by his/her mind. As a result, the process of creation of any theory becomes very personal and possesses dependence with the point of view of a given philosopher who proposes a theory. Hence, some theories possess names of their creators (like Newton's theory of gravity, Einstein's theory of Relativity and etc.).

\section{Identification of the Regularities}

According the definition of theory given above each theory uses more or less regularities observable by this or that way. Different theories use deferent sets of regularities they explain, and every single theory cannot be used outside of the relevant set of regularities. Therefore, regularities (based on observable facts and events) can be used as a cornerstone of a theory. Suppose now that there are some known facts $F_{A}\left(\right.$ fact $A$ ), $F_{B}($ fact $B$ ), $\mathrm{F}_{\mathrm{C}}$ (fact $\mathrm{C}$ ) and $\mathrm{F}_{\mathrm{D}}$ (fact $\mathrm{D}$ ). There are also three philosophers (J, K and L) who like to understand any regularity based on those facts. Each of them produced some analysis. As a result, they "detected" some regularity in the given scope of facts. Philosopher J "detected" regularity $\mathrm{R}_{\mathrm{J}}$ based on facts $\mathrm{F}_{\mathrm{A}}$ and $\mathrm{F}_{\mathrm{B}}$,

$\mathrm{R}_{\mathrm{J}}\left(\mathrm{F}_{\mathrm{A}}, \mathrm{F}_{\mathrm{B}}\right)$

Philosopher $\mathrm{K}$ “detected” regularity $\mathrm{R}_{\mathrm{K}}$ based on facts $\mathrm{F}_{\mathrm{B}}$ and $\mathrm{F}_{\mathrm{C}}$,

$\mathrm{R}_{\mathrm{K}}\left(\mathrm{F}_{\mathrm{B}}, \mathrm{F}_{\mathrm{C}}\right)$ 
And philosopher $\mathrm{L}$ "detected" regularity $\mathrm{R}_{\mathrm{L}}$ based on facts $\mathrm{F}_{\mathrm{A}}$ and $\mathrm{F}_{\mathrm{C}}$,

$\mathrm{R}_{\mathrm{L}}\left(\mathrm{F}_{\mathrm{A}}, \mathrm{F}_{\mathrm{C}}\right)$

Subsequently, those philosophers proposed some theory $\mathrm{N}\left(\mathrm{T}_{\mathrm{N}}\right)$ based on detected regularities $\mathrm{R}_{\mathrm{J}}$ and $\mathrm{R}_{\mathrm{K}}$,

$\mathrm{T}_{\mathrm{N}}\left(\mathrm{R}_{\mathrm{J}}, \mathrm{R}_{\mathrm{K}} ; \mathrm{F}_{\mathrm{A}}, \mathrm{F}_{\mathrm{B}}, \mathrm{F}_{\mathrm{C}}\right)$

And theory $\mathrm{O}\left(\mathrm{T}_{\mathrm{O}}\right)$, based on regularity $\mathrm{R}_{\mathrm{L}}$ and facts $\mathrm{F}_{\mathrm{A}}$ and $\mathrm{F}_{\mathrm{C}}$.

$\mathrm{T}_{0}\left(\mathrm{R}_{\mathrm{L}} ; \mathrm{F}_{\mathrm{A}}, \mathrm{F}_{\mathrm{C}}\right)$

However, no one of them was able to produce any theory that incorporates all known facts $F_{A}-F_{C}$ and propose a theory $\mathrm{P}\left(\mathrm{T}_{\mathrm{P}}\right)$ relevant to all known facts and detected regularities.

$T_{P}\left(R_{J}, R_{K}, R_{L} ; F_{A}, F_{B}, F_{C}\right)$

Moreover, no one of them noticed any regularity with fact $\mathrm{D}\left(\mathrm{F}_{\mathrm{D}}\right)$ and that fact was left without attention. As a result, $\mathrm{N}$-Theory, produced on facts and regularities mentioned above, possess the embedded problem. That is the irrelevance of the theory with the known fact $\mathrm{D}\left(\mathrm{F}_{\mathrm{D}}\right)$. Therefore,

Each theory depends on the facts and regularities which were put into considerations at the time of the creation of the theory and some facts and regularities which were rejected

The process of separation the "right" and "wrong" regularities from each other is very personal too and highly depends on the personal experience and the individual point of view of the philosopher (researcher). In other words, there is some "Logical Separator" (LS) hidden deep in the human mind that separates "possible" and "impossible" regularities. Under common circumstances, a person rare understands the presence of such thing in his/her mind. However, a person usually gives a certain answer on any question about possibility of a given regularity. Each regularity that can "go through" the Logical Separator looks like a possible one from the person's point of view. Otherwise, the regularity seems as "impossible" one. Strictly speaking, Logical Separator depends on some philosophical system that the person uses. As a result, each philosophical system establishes its own Logical Separator that "protects" the system from any regularity that seems "impossible" in the logical frame of a given philosophical system. Such situation leads to the most dramatic result that affects every attempt to use a new regularity or create a new theory.

The Logical Separator based on the "generally accepted" philosophical system rejects a newly detected regularity as "impossible" one and denies any attempt of further analysis of that regularity

Deep antagonism between an "unusual" regularity (and the fact) and a "generally accepted" philosophical system leads to often rejection of such regularities and facts as "impossible". They commonly make statement that those regularities "impossible" by themselves. However, "a real impossibility" appears only in the human mind as soon as regularity meets Logical Separator and becomes rejected by it. Such separation leads to one important side-effect. A philosophical system accepts regularities and facts only for some extent that cannot be expanded by the system. The most distant regularities and facts that keep compatibility with the philosophical system establish the Comprehension Horizon (of that system and a person who shares the system). In other words, anything inside the Comprehension Horizon seems "logical, good and well". Anything outside of the Comprehension Horizon seems "illogical, strange and mysterious". As result, any additional regularity inside the Comprehension Horizon leads to growth of a Philosophical System until appearance of a regularity that stays beyond the Comprehension Horizon and becomes incompatible with the established Philosophical System. Unfortunately, those regularities commonly treated as "mysteries" instead of close physical and philosophical examination. In that case, they can be used as a basis of new and more sophisticated Philosophical System. The best historical example of such transformation is a process of changes the human imagination from a Philosophical System based on the idea of immovable flat Earth to a 
new Philosophical System based on the idea of spherical Earth moving by its space trajectory (orbit) around the Sun.

Motion and Transposition: In case of Z-theory, the situation was the same. There were some "mysteries" before publication of Z-Theory that were so strange that it looks impossible to recognize any regularity based on those "mysteries". All of them were facts and observations beyond the Comprehension Horizon of any given theory existed at the time of observation. The best example of such phenomena was Boeing 727 incident. As a matter of fact, the ordinary aircraft was undetectable for the airport ground radar for 10 minutes during its descending by glide path. During that time, all attempts to reach the aircraft by radio have failed. After 10 minutes the aircraft repapered again and have made successful landing. But as it was found later all on-board timepieces indicated 10 minutes left from the indication of the airport clocks. Moreover, nobody onboard had noticed anything strange or unusual (Zade, 2012a). Subsequently, there was not any investigator who was able to produce any suggestion about the incident. The core problem was that. The Philosophical System accepted at the time of the incident based on the ideas of endless existence of an object in Space and endless flow of so-called "Time". Those core ideas and Logical Separator based on them deny any possibility of existence of any object that "jumps" from one point of space (and/or "Time") to any other point of "Space-Time" under any circumstances. However, the incident becomes analyzable in relationship with higher implementation of motion.

From the one hand, the incident taken from the Earth-bound observer looks like some "jump through Time". From the other hand, the same incident, taken from the point of view of a Sun-bound observer (who uses a Sun-bound reference frame) looks like "jump through Space". In a Sun-bound reference frame, the incident takes the following steps. There was some point "A" where an aircraft coexisted with the Earth. Suddenly the aircraft became "undetectable and unreachable by any means" (disappeared). The Earth continued its common orbital motion despite the incident. 10 minutes later, the point above the Earth surface, where the incident had palace, reached the point "B" (in the Sun-bound reference frame). Suddenly, the same aircraft reappeared at the point "B". Therefore, the incident looks like Space jump from a Sun-bound observer's point of view (in the Sun-bound reference frame). Moreover, as soon as the same aircraft used the same points "A" and "B", those points should be connected by some sort of relationship (by a possible trajectory in common understanding) that caused a "strange, undetectable" relocation of the aircraft. Later, that trajectory possessed a specific name of Z-Trajectory. The noticeable aspect of the entire event was that. The aircraft had the same altitude before and after "disappearance" in complete coincidence with the law of conservation. Subsequently, that law was extended to the entire theory, and many phenomena were explained by application of the same law.

By definition given in the book: Z-Trajectory is a special part of a moving object's trajectory that cannot be viewed or interacted with by the IB (Independent Bystander) and always lies between two special points where the difference in the whole energy of moving object equals zero. As a result, the whole energy of a system keeps constant before and after an object uses Z-Trajectory. In other words Z-Trajectory is the image trajectory that connects two points of space with equal value of full strength of conservative fields by means of a special number of points that have no interaction with the surrounding conservative fields produced by independent bodies (Zade, 2011, p. 240). Subsequently, motion of an object by Z-Trajectory became one of ZTheory's cornerstones and possessed a specific name of Transposition to be distinct from common way of Motion in common Space. Later, that distinction was explained in details in the article "Motion and Transposition in conservative fields" (Zade, 2012b).

A Matter of Time: Everybody who tries to think about Time turns immediately to nonplus. The core problem of that situation is this. There was not any suitable definition of Time before 2012. As a result, there was no way to think about anything that has not any recognizable set of attributes. Therefore, as long as there is not any definition of so-called "Time" there is not any way to make any conclusion about any category related to "Time". There is not also any possibility to make "Right" or "Wrong" decision about anything related with "Time". As a result, everything that the humankind "knew" about so-called "Time" falls under question. The core illusion was that. According to the established Philosophical System, "Time" appears as some aspect of physical reality of the Universe because it seems "measurable by clocks". However, there is not any clock that can make "measurement of Time" by itself. The easiest example for that is this. There is not any clock that can 
make "anticipated indication" after interruption. Suppose, someone made interruption of operation of a clock. In that case:

- the clock is unable to go back to operation by itself

- the clock after restoration of its operation by a man keeps some difference in indication in comparison with a clock that had not any interruption

- the interrupted clock shows not any intention to "sped up" its indications (after restoration of its operation) to "match" indications with the uninterrupted clock

Those aspects of the way of operation completely mismatch operation of other real measurement devices like thermometers because every thermometer makes measurement of a real physical aspect of an object (substance). As a result, two thermometers ever show the same indications by themselves under same circumstances without any interaction with a human being. That aspect of clock operation was described in the chapter 12.7 "Operation of Watches and Clocks" of the book (Zade, 2011, p. 102-105). Later, the same point of view was explained in details in the article "Human's Delusion of Time". The article gives the exact answer on the question about nature of Time. According to the article we have the following aspects of socalled "Time":

\section{What is Time?}

Logical Definition: Time is a logical link in human mind to any physical process that has observable duration.

Physical Definition: Time does not exist (and never existed) as a physical property of the Universe.

Mathematical Definition: Time means a rate of durations between any two different physical processes.

Philosophical Definition: Time is ancient innate idea of humankind.

Common Definition: Time is a link between indication of a clock and duration of its own internal recurrent physical process.

What is "Now"?

"Now" is a point in the Universe from where an observer (object, body, etc.) makes interaction with surrounding Universe (Zade, 2012d).

The clear physical definition mentioned in the citation given above created the next cornerstone of Z-Theory.

The Matter of Navigation: Many phenomena mentioned above create a significant problem of navigation for every man-made vehicle involved in motion on Z-Trajectory. The result of Transposition usually appears as sudden "mysterious relocation" of a vehicle from one location to another one. The next example of Transposition comes from the experience of Bruce Gernon, who was involved in Transposition with an aircraft more than once. Later, Mr. Gernon made a significant effort to explain those incidents and proposed his point of view on that matter in the book "The Fog" (Gernon, 2005). Strictly speaking, Mr. Gernon described some phenomenon that caused "mysterious relocation" of his A-36 aircraft. According to information from Bruce his aircraft had air-journey for 250 miles in 47 minutes flying from Andros Island Town Airport to West Palm Beach Airport and had successful landing with 9 extra gallons of unspent fuel (Zade, 2012a). Moreover, Mr. Gernon described some unusual optical phenomenon he experienced during the flight.

In that case, A-36 used some Z-Trajectory as well as Boeing 727. The difference was that. In the second case the planet didn't move during relocation of the aircraft in comparison with the aircraft relocation. As a result, A-36 appeared at the end point of Z-Trajectory almost immediately after "disappearance" from the first point 
of Z-Trajectory. The duration of relocation by Z-Trajectory for A-36 and Boeing 727 was the same and equal almost to zero in both cases. The difference appeared only for Earth-bound observers. They did not notice any difference in indications of onboard clock and Earth-bound clocks in the second case. In other words, duration of A-36 Transposition and Motion of the planet during Transposition were the same. As a result, both sets of clocks onboard and Erath-bound count the same duration of their internal recurrent processes. Hence, indications of those clocks were the same before and after the Transposition in that case. The same aspect of operation of a clock (or a watch) caused the age-old problem for the human mind. Any couple of timepiece count only oscillations of their own recurrent physical process and nothing more. Durations of those processes are the same ever for any number of Earth-bound timepieces. As a result, any numbers of previously synchronized Earth-bound timepieces show the same indications as long as they keep identical trajectories in the common space.

That aspect caused formulation of the idea of so-called "Time" as some "mysterious connection" between all timepieces. Bruce also noticed coincidence in indications between the onboard clock of his aircraft and Earthbound clocks. Taking that fact as distinguish between Boeing 727 incident and his experience he rejected the idea of any similarity in both incidents. He tried to make an explanation of his experience be himself and created later an idea of something that he called "Electronic Fog." According the idea of Electronic Fog, A-36 aircraft was swollen and dragged many miles away from the aircraft original location (by Electronic Fog). That is the best example of two different and incompatible regularities built on the same set of facts. From the one hand, Z-Theory offers notion of Z-Trajectory. From the other hand, Bruce Gernon offers notion of Electronic Fog. The key difference between Z-Theory and the idea of Electronic Fog is this. Z-Theory uses ZTrajectory to explain the phenomenon of Transposition. Z-Trajectory connects two specific points of Space but exists outside of something that we call Space. Unlike Z-Trajectory, Electronic Fog keeps its operation only inside Space. That happened because an idea of something that can exist outside of Space was immediately rejected by Bruce's Logical Separator (possibly at the level of unconsciousness).

Moreover, Bruce mentioned by himself something that could not be explained even by his idea of Electronic Fog. That is something that Bruce described as "Yellow Fog". Bruce experienced that thing surrounded his aircraft just before the aircraft left "The Fog". In other words, "Yellow Fog" as a matter of fact stays beyond the Comprehension Horizon of "The Electronic Fog Theory" created by Bruce himself. Unlike "The Electronic Fog Theory" (EFT), Z-Theory spreads its Comprehension Horizon far beyond that phenomenon and uses it as a consistent part of the theory. According Z-Theory, "Yellow Fog" or "Colored Fog", in general case, appears as a combination of two phenomena called Fog Effect and Rainbow Effect. Both phenomena described and explained in details in the book in chapters 12.1 "Cloud Mistake" (Zade, 2011, p. 86-87) and 12.2 "Rainbow Effect" (Zade, 2011, p. 87-91). As a result an IO (Independent Observer) watches some coloration with vision covered by some fog (Zade, 2011, p. 91). There is also one more phenomenon noticed by Bruce. His A-36 aircraft consumed not some fuel necessary to reach West Palm Beach Airport, and Bruce detected some extra fuel in the gas tank of the aircraft at the point of destination. That coincides with the idea of Transposition that reduces the duration of the aircraft flight. Hence, an aircraft spends lesser volume of fuel than expected.

Transposition is also responsible for one more "mystery" known as "Early arrival Phenomenon". That phenomenon appears ever as soon as the aircraft reaches its point of destination earlier than expected and calculated speed of the aircraft becomes "impossible" for a given aircraft type. Any known physical process like "strong tail wind" cannot explain that phenomenon. Such "explanations" become irrelevant to the problem because modern commercial aircrafts fly at speed near the speed of sound. Any significant attempt to increase the cruising speed of such aircraft leads to physical destruction of the aircraft because its entire frame did not match requirement for supersonic flight. For example, a commercial aircraft takes a route with 6 hours long flight. But, suddenly, the aircraft reaches the point of destination within only 3 hours. The calculated speed for such "flight" gives value about Mach 2 (twice the speed of sound). Zade, (2012a) gives a little restoration of such event.

The Matter of Imagination: There should be some similarity at the first and the last points of Z-Trajectory to make Transposition possible. Otherwise, much regularity should not be detected (like equality of altitude of aircrafts before and after Transposition). To match that regularity, there should be something that exists regardless of an object that uses Z-trajectory and keeps the same condition regardless any possible duration 
of the process of physical Transposition. There is only one thing known in physics that meets such requirements. That is a conservative field. There are two sorts of conservative fields known today. Those are gravitational field and electric field. Therefore, all phenomena relevant to Z-Theory should be relevant to those fields also. The general law applicable to the first and the last points of Z-Trajectory (Head Points of ZTrajectory) seems quite easy:

$$
\left|\mathrm{S}_{\mathrm{CA}}\right|=\left|\mathrm{S}_{\mathrm{CB}}\right|
$$

To make Transposition possible between two points $A$ and B, magnitude of Strength of a conservative field at both points should be equal.

Physical application of the equation (7) and statement (I) leads to the location of a man-made aircraft at the same true altitude (above the sea level) before and after Transposition in both incidents mentioned above (Boeing 727 and A-36). The relationship of transposition with true altitude instead of absolute altitude (distance above land or water) leads to some phenomena that stay far beyond the Comprehension Horizon of other theories. Suppose now that a Z-Trajectory becomes possible between two points A and B. Moreover, point $A$ appears in a stream running down the mountain. There is also a school of fish going down the stream. The school of fish meets the Z-Trajectory Head Point at the point A and makes Transposition to the point $\mathrm{B}$. Everything looks fine that way, but the point B can be located far away from the point A. Hence, it is quite possible that there is not any river at the point B. Moreover, the point can be located in mid-air if the Erath surface at the point locates lower than at the point A. In that case, the school of fish that appeared in mid-air follows the universal law of gravitation and falls down the Earth as well as any other object located in the gravitational field of the Earth. Is it possible physically? There is a positive theoretical answer on that question given in the book in the Chapter 13 "Biological Appearances" (Zade, 2011, p. 126 - 139). There is also a physical example of the same phenomenon coming from Lajamanu, Australia.

"NEWSBREAKER Christine Balmer ... had to pinch herself when she was told "hundreds and hundreds" of small white fish had fallen from the sky.

"It rained fish in Lajamanu on Thursday and Friday night," she said, "They fell from the sky everywhere.

"Locals were picking them up off the footy oval and on the ground everywhere.

"These fish were alive when they hit the ground" (Zade, 2012c)

Therefore, all those phenomena are only conservative field phenomena and have not any relationship with aerial or naval phenomena.

\section{Conclusion}

Z-Theory looks like something that "cannot be understood ever" for a lot of people. The core problem for such point of view is this. Any attempt to understand Z-Theory by categories of 20-th-century philosophy and physics fails completely because Z-Theory uses a different theoretical frame based on a different core described above to some extent. The most significant difference between Z-Theory and other earlier appeared theories is this. The theory uses only theoretically clear categories with detailed descriptions. All those categories appear in Z-Theory along with exact definitions and sets of attributes. That way helps to change many categories from unthinkable to thinkable and understandable by their attributes. Z-Theory also uses not any uncertain categories like postulates and anything derived from them. Many people believe that the best advantage of Z-Theory is absence of so-called Time in the theoretical frame. However, that is only one noticeable aspect of Z-Theory. That aspect helps to reach solutions of many long lasting problems in philosophy and physics taken with other aspects of the theory.

\section{References}

Gernon B. (2005). The Fog: A Never Before Published Theory of the Bermuda Triangle Phenomenon. Llewellyn Publications. ISBN 978-0738707570

Scientific Theory. (2008). Encyclopædia Britannica. Encyclopaedia Britannica 2008 Deluxe Edition. Chicago: Encyclopædia Britannica.

Zade A. (2011). Z-Theory and Its Applications. AuthorHouse. ISBN 978-1452018935 
Zade A. (2012a). September). Matter of Navigation. International Journal of Scientific and Research Publications (IJSRP), 2(9).

Zade A. (2012b). Motion and Transposition in conservative fields. International Journal of Scientific and Research Publications (IJSRP), 2(8).

Zade A. (2012c). Gravitational fields and Transposition in Australian authenticated case. International Journal of Scientific and Research Publications (IJSRP), 2(10).

Zade A. (2012d). Human's Delusion of Time. International Journal of Scientific and Research Publications (IJSRP), 2(10). 